\title{
Effect of Helicobacter pylori Infection on the Composition of Gastric Microbiota in the Development of Gastric Cancer
}

\author{
Lei Cao Jun Yu
}

Institute of Digestive Disease and Department of Medicine and Therapeutics, State Key Laboratory of Digestive Disease, Li Ka Shing Institute of Health Sciences, CUHK-Shenzhen Research Institute, The Chinese University of Hong Kong, Shatin, Hong Kong, SAR, China

\author{
Key Words \\ Gastric carcinogenesis · Gastric microbiota · Helicobacter pylori
}

\begin{abstract}
Background: Gastric cancer is one of the most common cancer types worldwide. In China, gastric cancer has become one of the major threats for public health, ranking second on incidence and third on cause of cancer death. Despite the common risk factors that promote the development of gastric cancer, the huge quantity of microorganism colonies within the gastrointestinal tract, particularly Helicobacter pylori infection, demonstrates a correlation with chronic inflammation and gastric carcinogenesis, as epidemiological studies have determined that $H$. pylori infection confers approximately $75 \%$ of the attributable risk for gastric cancer. Summary: The current article draws an overview on the correlation between the microbiota, inflammation and gastric tumorigenesis. $H$. pylori infection has been identified as the main risk factor as it triggers epithelial barrier disruption, survival signaling as well as genetic/epigenetic modulation. Apart from $\mathrm{H}$. pylori, the existence of a diverse and complex composition of microbiota in the stomach has been identified, which supports a role of microbiota in the development of gastric cancer. Moreover, metagenomics studies focused on the composition and function of the microbiota have associated microbiota with gastric metabolic diseases and even tumorigenesis. Apart from the gastric microbiota, inflammation is another identified contributor to cancer development as well. Key Message: Though H. pylori infection and the non- $H$. pylori microbiota play a role in gastric cancer, the properties of gastric microbiota and mechanisms by which they participate in the genesis of gastric cancer
\end{abstract}


are still not clearly depicted. Moreover, it remains to be understood how the presence of microbiota along with $H$. pylori infection affects the progress from gastric disease to cancer. Practical Implications: This article summarized a clue of the current studies on microbiota, H. pylori infection and the progression from gastric disease to cancer. @ $2015 \mathrm{~S}$. Karger AG, Basel

\section{Introduction}

Stomach cancer still remains the most common cancer burden in the world. Aged people are at high risk for gastric cancer, as most people diagnosed with stomach cancer are between their late 60s and 80s. Despite a major decline in incidence and mortality, it is estimated that in 2012 there were 951,594 people diagnosed with gastric cancer and about 723,027 died from this disease, making it the fifth cause of new cancer cases and the third cause of cancer mortality [1]. When comparing among nations, the incidence of gastric cancer is particularly high in Japan, China, Southern and Eastern Europe as well as South and Central America, whereas the lowest rates are observed in North America and most parts of Africa [2]. In China, the incidence of gastric cancer ranks second among all cancer types for both sexes, while it ranks third in men after lung and liver cancer and in women after breast and lung cancer [3]. The world-adjusted mortality rate for gastric cancer in China is the highest worldwide, both for men and women (table 1) [4], with the highest mortality geographically occurring in the northern and mid-western provinces. Comparing with the urban areas in China, the rural areas, especially in Gansu, Henan, Hebei, Shanxi and Shaanxi Provinces in the mid-western part of China, present a high risk [5]. While the etiology of gastric cancer is multifactorial and yet to be fully understood, a number of important risk factors have been revealed, including older age, male sex, Helicobacter pylori infection, tobacco smoking, diet and family history [6].

H. pylori is a Gram-negative bacterial species that specifically colonizes the gastric epithelium. Most people are colonized by $H$. pylori and hence develop co-existing gastritis for decades. Previous studies provide evidence that $H$. pylori co-evolved with humans at least since their migration out of East Africa nearly 60,000 year ago [7]. H. pylori is hence regarded as a component of the human normal gastric microbiota. Ever since the first discovery of H. pylori in the early 1980s by Dr. Barry J. Marshall and Dr. J. Robin Warren [8], H. pylori has been identified as the main cause of chronic gastritis and peptic ulcer [9]. Depending on the prevalence of $H$. pylori, the attributable risk of gastric cancer conferred by $H$. pylori increases from $75 \%$ to $>90 \%$ [10]. H. pylori infection is hence the strongest known risk factor for gastric cancer when compared with other ones. For decades, researchers have dedicated their efforts to depicting the mechanisms modulating the biological interactions between $H$. pylori and its hosts and whether it contributes to carcinogenesis.

Apart from $H$. pylori, the stomach harbors $10^{3}-10^{4}$ bacteria, and this huge number of microorganisms can cause pathology under some condition, such as immunodeficiency [11] or microbiota dysbiosis. Bacteria from the upper digestive track, respiratory tract and small intestine can enter the stomach, as the stomach is in the central position that connects the esophagus and oral cavity on the upper side and the duodenum on the lower side [12]. Though its low-pH environment was believed unsuitable for bacterial colonization, the existence of acid-resistant bacterial strains was confirmed by previous studies [13]. With the application of high-throughput sequencing technology in microbiology, the existence of huge numbers of bacteria other than H. pylori in the stomach was proved and a more detailed stomach flora constitution was obtained.

However, few studies on the gastric microbiota have been performed to fully understand the constitution and diversity of the microbiota under health and disease situations as well 
Cao and Yu: Effect of Helicobacter pylori Infection on the Composition of Gastric Microbiota in the Development of Gastric Cancer

as the function of microbiota in the pathogenesis of various digestive diseases. Therefore, the current article provides an overview of the recent findings regarding the relationship between gastric microbiota, microenvironment and gastric tumorigenesis.

\section{Community Composition and Biodiversity of Gastric Microbiota}

For a long time, the stomach was believed unsuitable for bacteria colonization. Due to the limitations of traditional culture-based methods, early microbiological studies failed to demonstrate the overall microbial community structure, which includes the uncultured microorganisms. The major risk factor for gastric cancer, H. pylori, was isolated from the stomach by Marshall et al. in 1982 [8]. With the application of metagenomics and highthroughput sequencing technology in microbiology, the identification of the stomach flora has increased. Although the composition of the stomach microbiota has not been clearly profiled and documented, 130-260 phylotypes from up to 13 phyla have been detected [1416]. On the phylum level, Proteobacteria (to which $H$. pylori belongs) seems to be the most abundant in the normal gastric microbiome, quickly followed by Firmicutes and then by Bacteroidetes, Actinobacteria and Fusobacteria. The Proteobacteria mainly consists of Helicobacter, the genera Haemophilus, Actinobacillus and Neisseria. Firmicutes is mainly composed of species from the genera Streptococcus and Bacillus, while Prevotella species contribute to the dominance of Bacteroidetes. Rothia,Actinomyces and Micrococcus species are the dominant Actinobacteria [14-17]. Later, Delgado et al. [18] analyzed stomach mucosa biopsies of 12 healthy persons and confirmed that Firmicutes and Proteobacteria were the most abundant phyla followed by members of the phylum Actinobacteria, while Deinococcus-Thermus, Bacteroidetes and Gemmatimonadetes were represented in small numbers $(<3 \%)$. The sequences were grouped into 59 families (represented by Streptococcaceae, Lactobacillaceae and Propionibacteriaceae) and 69 genera, revealing wide bacterial diversity. Among all, 19 genera were found to compose $35-54 \%$ of the total genera detected per sample, including Lactobacillus, Lactococcus, Propionibacterium, Staphylococcus, Streptococcus and Brevibacterium, Methylobacterium, Pseudomonas, Serratia, Stenotrophomonas, Veillonella and Vibrio bacteria (fig. 1).

\section{Differences in Microbiota Composition between Cancer and Non-Cancer Tissue}

Although what represents normal microbiota in the stomach has not been well established, differences in microbiota composition have been detected between patients suffering from digestive diseases and healthy subjects as well as among groups of healthy human subjects. Concerning variations in the microbiota, Aviles-Jimenez et al. [19] found that bacterial diversity showed a trend to diminish from non-atrophic gastritis to intestinal metaplasia and to gastric cancer, with a decrease in Porphyromonas, Neisseria, TM7 group and Streptococcus sinensis as well as an increase in Lactobacillus coleohominis and Lachnospiraceae, which might favor development of gastric cancer. In contrast to previous findings that in the gastric mucosa the most abundant families are Streptococcaceae and Prevotellaceae, Aviles-Jimenez et al. detected Lachnospiraceae as the most abundant (representing almost $20 \%$ of the microbiota) in all patients by G3 PhyloChip ${ }^{\mathrm{TM}}$. Additionally, the genus Pseudomonas is one of the taxa that were significantly more abundant in gastric cancer than in nonatrophic gastritis. The analysis of microbial communities between normal and paired cancer samples from given $H$. pylori carriers displayed that normal gastric mucosa had larger populations of Propionibacterium spp., Staphylococcus spp. and Corynebacterium spp. than that of 
Cao and Yu: Effect of Helicobacter pylori Infection on the Composition of Gastric

Fig. 1. The microbiota composition in normal stomach [14-18]. The dominant genera are highlighted in red.

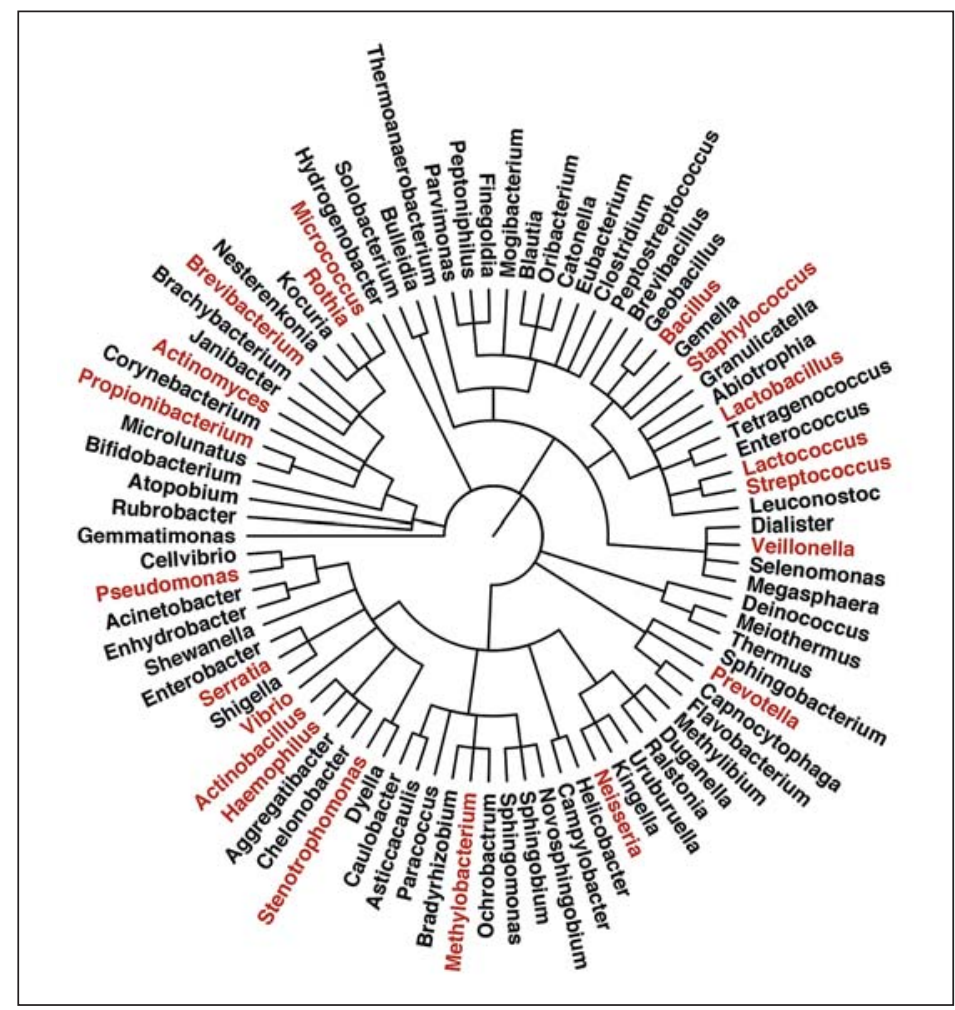

Table 1. Estimated incidence and mortality of stomach cancer worldwide in 2012 [4] (estimated numbers, $\times 1,000)$

\begin{tabular}{|c|c|c|c|c|c|c|}
\hline & \multicolumn{2}{|l|}{ Men } & \multicolumn{2}{|c|}{ Women } & \multicolumn{2}{|c|}{ Both sexes } \\
\hline & cases & deaths & cases & deaths & cases & deaths \\
\hline World & 631 & 469 & 320 & 254 & 925 & 723 \\
\hline More developed regions & 175 & 107 & 99 & 68 & 275 & 175 \\
\hline Less developed regions & 465 & 362 & 21 & 186 & 677 & 548 \\
\hline United States & 13 & 7 & 8 & 5 & 21 & 12 \\
\hline European Union (EU-28) & 51 & 35 & 31 & 23 & 82 & 58 \\
\hline China & 283 & 221 & 419 & 104 & 405 & 325 \\
\hline India & 43 & 41 & 31 & 18 & 63 & 59 \\
\hline
\end{tabular}

gastric cancer, while Clostridium and Prevotella were denser in gastric cancer mucosa than in normal mucosa of the stomach [17].

The microbial composition analysis of gastric mucosa from patients with chronic gastritis, intestinal metaplasia and gastric cancer demonstrated that Epsilonproteobacteria (containing H. pylori) appeared to be the most prevalent and Epsilonproteobacteria in most of the enrolled patients, especially in $\mathrm{H}$. pylori-positive patients by conventional methods, were predominantly composed of $H$. pylori species $[16,20,21]$. Thus, $H$. pylori is the most dominant organism, representing $>50 \%$ of all bacterial cells in the human stomach in $H$. pylori-positive subjects when detected by conventional means, including culture or biochemical assays, tissue histology and host serological responses [16, 20, 21]. However, in the same patients carrying 
Cao and Yu: Effect of Helicobacter pylori Infection on the Composition of Gastric

Microbiota in the Development of Gastric Cancer

H. pylori, the microbial profile showed that the abundance of H. pylori in normal gastric mucosa was higher than that of the paired gastric cancer patients. This decrease in the $H$. pylori population in cancer tissue was associated with the hypochlorhydric mucosal environment, which is not suitable for $H$. pylori colonization [17].

\section{Impact of $\boldsymbol{H}$. pylori on Gastric Microbiota}

Chronic H. pylori infection is likely to cause gastric microbiota shift due to the H. pyloriassociated changes in gastric physiology and immunology, e.g. reduced gastric acidity, perturbed nutrient availability and local innate responses [22]. In mouse models, the mouse genetic background, the strain of $H$. pylori and the $H$. pylori infection period may affect the ability of H. pylori to alter the gastric microbiota. Colonization by H. pylori in specific pathogenfree female BALB/c mice leads to reduced abundance of Lactobacillus species in the gastric microflora when compared with non-infected mice [23]. In the transgenic, insulin-gastrin (INS-GAS) mouse model, the H. pylori-infected specific pathogen-free male mice showed significant differences at the phylum level compared with the control mice, with marked reductions in Bacteroidetes and marked increases in Firmicutes [24]. In female C57BL/6N mice, H. pylori colonization included decreased Firmicutes (class Bacilli), Bacteroidetes and Proteobacteria and increased Firmicutes (class Clostridia), Proteobacteria (genus Helicobacter) and Verrucomicrobia, but did not substantially change the overall stomach microbiota composition [25]. Osaki et al. [26] determined the differences in the gastric microbiota between $H$. pylori-positive and -negative Mongolian gerbils, indicating that the number of Bifidobacterium spp. in H. pylori-positive gerbils increased compared to that in the H. pylorinegative group, while Eubacterium cylindroides and Prevotella spp. were detected only in H. pylori-negative gerbils.

There are also a limited number of studies reporting the effect of $H$. pylori on the microbiota of the human stomach. The gastric microbial profiles of $H$. pylori-positive patients recorded enriched relative abundances of non-Helicobacter bacteria from Proteobacteria, Spirochetes and Acidobacteria [27], specifically from the families Bradyhizobiaceae, Caulobacteraceae, Lactobacillaceae and Burkholderiaceae by comparison with $H$. pylori-negative patients. For H. pylori-negative patients, class Alpha-, Beta-, Gamma-Proteobacteria, Bacilli, Bacteroidia, Clostridia, Flavobacteria, Fusobacteria or Negativicutes were found as major taxa [20]. Another study demonstrated that patients positive for H. pylori culture showed significantly increased colonization of Proteobacteria and a decrease in Actinobacteria [19].

The above studies suggested that infection with $H$. pylori was closely correlated with the remaining stomach flora. However, due to the limitations of animal models and case studies, the role of the human stomach microbiota in intragastric colonization of $H$. pylori has not been comprehensively understood.

\section{Gastric Microbiota Alteration following Eradication of $\boldsymbol{H}$. pylori and Gastric Cancer Development}

Although eradication of $H$. pylori before the onset of atrophy demonstrated the possibility to protect against gastric cancer [28-30], the antibiotics used affected the commensal microbiota. The few studies that have been performed on gastrointestinal microbiota alteration following eradication of $H$. pylori were based on standard culture methods instead of the current high-throughput metagenomics approach. In one study, 14 patients received $20 \mathrm{mg}$ omeprazole, 1,000 mg amoxycillin plus $400 \mathrm{mg}$ metronidazole (OAM), while another 16 

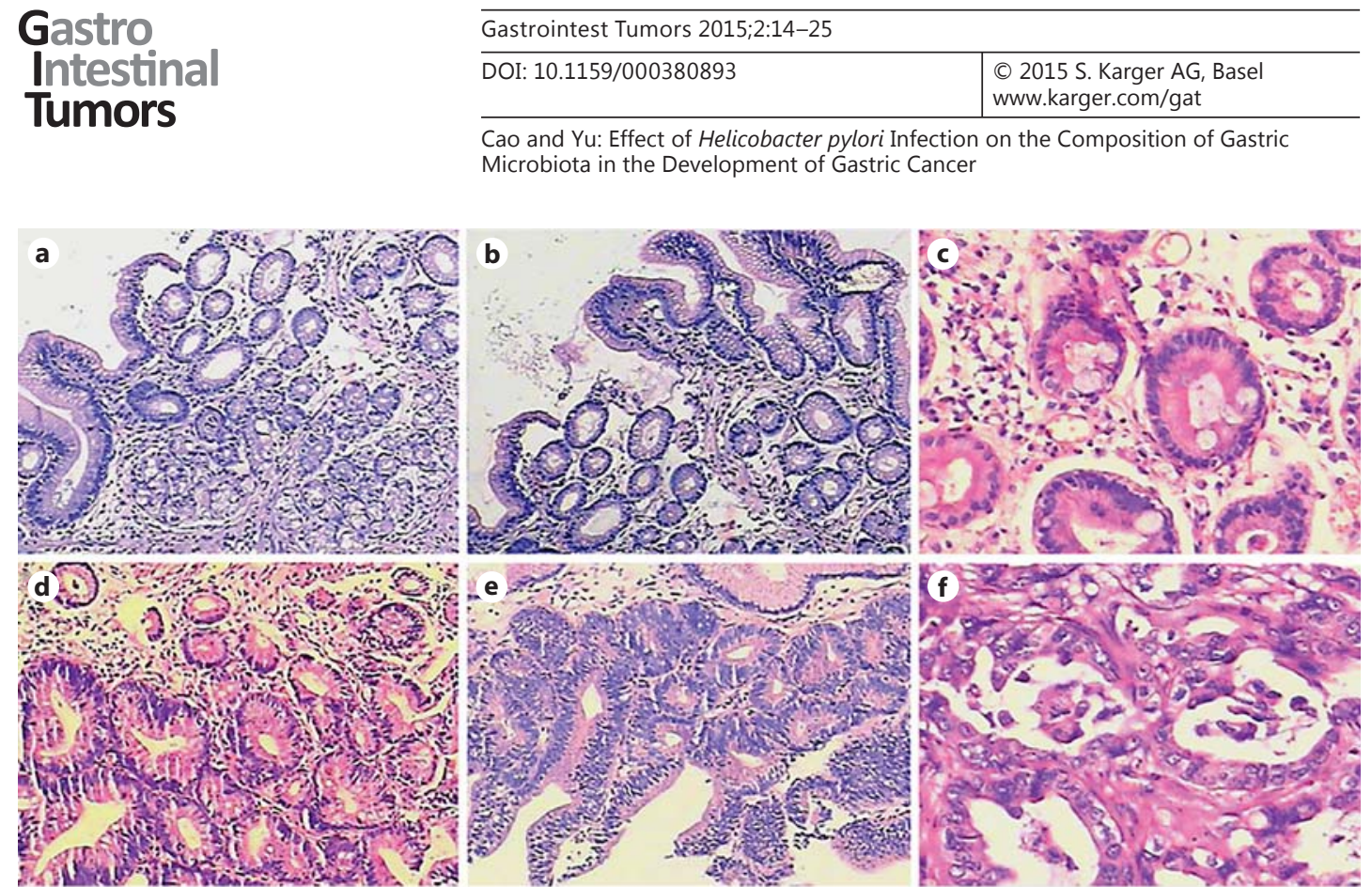

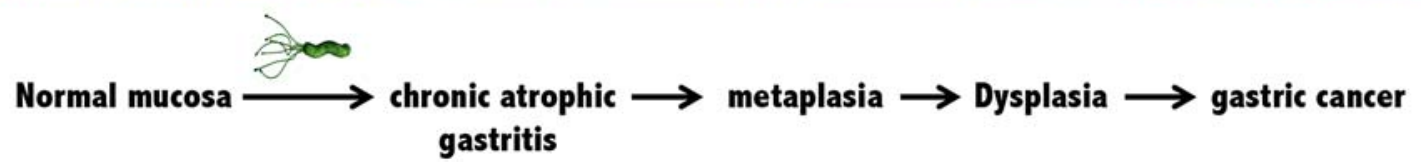

Fig. 2. The sequential histological events in the progression from superficial gastritis (a), atrophic gastritis (b), intestinal metaplasia (c), low-grade (d) and high-grade gastric dysplasia (e) to gastric adenocarcinoma (f) $[82]$.

patients were given $20 \mathrm{mg}$ omeprazole, $250 \mathrm{mg}$ clarithromycin plus $400 \mathrm{mg}$ metronidazole (OCM), both twice daily for 7 days. Concerning the intestinal microbiota, for both OAM and OCM treatment, there was also a significant increase in the numbers of Enterococci, Enterobacteria and Peptostreptococci. Anaerobic bacteria such as Bifidobacteria and Clostridia were significantly suppressed. These alterations returned to normal 4 weeks after treatment in the OAM group, but persisted in the OCM group [31]. Researchers in Germany observed a decreased colonization in non-spore-forming anaerobic bacteria and an increased colonization with yeasts including Candida albicans, and with Clostridia, after treatment by OCM on the intestinal microflora of 57 patients and 21 controls [32].

Due to the lack of experimental support and the limitation of previous studies, the way gastric microbiota shift and what role the microbiota alteration plays in gastric cancer development is yet to be explored.

\section{Roles of $\boldsymbol{H}$. pylori in the Development of Gastric Cancer}

Gastric cancer is classified as diffuse and intestinal type. The development of the intestinal type follows the sequential events from superficial gastritis to chronic atrophic gastritis to intestinal metaplasia, then to dysplasia and finally to gastric adenocarcinoma (fig. 2). During gastric carcinogenesis, genetic predisposition, infection and diet are identified as part of a complex interaction, among which the ongoing local chronic inflammatory induced by H. pylori is likely to be one of major factors for gastric lymphoma development. However, only a small percentage of colonized individuals develop clinically apparent sequelae, though all persons carrying $H$. pylori have coexisting gastric inflammation [33]. 
Cao and Yu: Effect of Helicobacter pylori Infection on the Composition of Gastric Microbiota in the Development of Gastric Cancer

\section{Molecular Mechanisms of Epithelial Barrier Disruption by H. pylori}

The majority of $H$. pylori exist as free-living microorganisms in the hosts, while there is about $20 \%$ binding to gastric epithelial cells, which are the first barrier against pathogenic microbes [34]. Thus, intimate interactions between $H$. pylori and epithelial cells are likely to contribute to pathogenesis. Studies have revealed that highly virulent $H$. pylori strains harbor pathogenicity factors, including Cag pathogenicity island, cytotoxin-associated gene A (CagA), vacuolating cytotoxin [35], OipA, peptidoglycan, iceA and several $H$. pylori-expressing adhesins. Cag pathogenicity island encodes a type 4 secretion system to inject the bacterial CagA.

In normal epithelium, intercellular junctions and interactions, including tight junctions (TJs), adherens junctions (AJs) and focal adhesions, exhibit tumor-suppressive and/or antimetastasis properties. CagA is key factor leading to chronic gastritis and ulceration, mucosaassociated lymphoid tissue lymphoma and gastric cancer in humans [34, 36-38]. Injected CagA causes depolarization and disruption of the tight junction barrier function in epithelial cells by recruiting the scaffolding protein ZO-1 to the H. pylori attachment sites [39-41] or by binding to Par1b to form the CagA-Par1b complex which mislocalizes to TJs and apical membranes [40, 42]. Massive injection of CagA into host cells results in the disruption of AJs, as the transfected CagA physically interacts with E-cadherin, a key molecule of AJs, impairing the interaction between E-cadherin and $\beta$-catenin and then the stability of the AJ complex [43]. CagAPY (tyrosine-phosphorylated at Glu-Pro-Ile-Tyr-Ala [EPIYA] motifs) translocates and can interact with Crk adaptor proteins to form the CagAPY-Crk complex, which stimulates the breakdown of AJs [44]. Apart from CagA, the highly virulent H. pylori strains also harbor VacA, which is secreted by the bacteria and binds to receptors RPTP $\alpha$ and RPTP $\beta$ (receptor protein tyrosine phosphatase $\alpha$ and $\beta$ ) on epithelial cells. After binding to RPTP $\alpha$ and RPTP $\beta$, VacA induces pores and vacuoles, along with the occurrence of VacA internalization [45]. BabA and BabB, SabA as well as AlpA and AlpB are important adhesins, secreted by $H$. pylori for establishing host-cell contact [46]. As soluble factors, VacA and adhesins could directly open TJs and AJs in a mild way via activation of cellular factors [47]. Cytotoxin-associated gene L (CagL), a specialized adhesin on the type 4 secretion system pilus, binds to and activates integrins, which trigger the delivery of CagA across the host-cell membrane [48].

H. pylori has also been reported to trigger various pathways in the host cell after adherence, including JAK/STAT3, NF- $\mathrm{KB}, \mathrm{PI} 3 \mathrm{~K} / \mathrm{Akt}$, Wnt/ $\beta$-catenin and Ras/Erk, thus inducing carcinogenesis. As mentioned previously, CagA, the virulence factor of $H$. pylori, can be injected into the host cells following the activation of the signaling cascades for tumorigenesis. Once translocated into epithelial cells, CagA is phosphorylated by members of the Src family of kinases at the Glu-Pro-Ile-Tyr-Ala (EPIYA) motifs [49-52]. CagL was also reported to activate FAK and Src early in $H$. pylori infection to guarantee phosphorylation of CagA directly at the injection site [48]. Phospho-CagA subsequently activates a eukaryotic phosphatase (SHP-2) as well as ERK, a member of the MAPK family, leading to elevation of expression of a newly identified oncoprotein CIP2A in AGS cells [53, 54]. Moreover, H. pylori cag+ strains activate heparinbinding EGF in vitro, thus triggering the transactivation of EGF receptor and subsequently inducing the early growth response gene Egr-1 via Ras-mediated activation of ERK1/2 [55, 56]. However, the EGF receptor has also been demonstrated to be activated by the cag-independent way, in which bacterial $\gamma$-glutamyltranspeptidase has been identified as a potential upregulator of heparin-binding EGF in gastric epithelial cells [57]. Additionally, deletion of H. pylori OipA affected the phosphorylation status of FAK [58].

The transactivation of epidermal growth factor receptor (EGFR) [59] by $H$. pylori also induces PI3K/AKT cascade activation in gastric epithelial cells. Upon ligand stimulation, EGFR forms dimers and phosphorylates PI3K, leading to activation of the PI3K/AKT pathway and $\beta$-catenin nuclear localization [56, 60, 61]. Transactivation of EGFR induced by H. pylori has also been shown to enhance PI3K/AKT signaling in a Src-dependent manner, which 
involved the cag secretion system and peptidoglycan in H. pylori [60]. The CRPIA motif in nonphosphorylated CagA was involved in interacting with activated Met, leading to sustained activation of PI3K/Akt signaling in response to H. pylori infection [62]. In addition to CagA, the type 4 bacterial secretion system also injects $H$. pylori peptidoglycan into gastric epithelial cells, resulting in activation of the PI3K pathway [63]. VacA was reported to induce activation of the PI3K/Akt signaling pathway and subsequent inhibition of GSK3 $\beta$ when incubated with AZ-521 cells in vitro [64].

Once phosphorylated by AKT, GSK3 $\beta$ gets inactivated and then releases $\beta$-catenin, a downstream component of the Wnt signal transduction pathway, for nuclear translocation. Apart from CagA, H. pylori constituents such as VacA and the adhesion molecule OipA have been proved to mediate $\beta$-catenin nuclear localization. VacA translocates the $\beta$-catenin into the nucleus by inactivating GSK3 $\beta$ [64], while the inactivation of OipA decreases $\beta$-catenin nuclear localization [65].

\section{H. pylori and Genetics/Epigenetics}

H. pylori infection mediates epigenetic regulations in the gastric epithelium, consequently causing gastric carcinogenesis. It has been reported that some miRNAs expression levels varied significantly between $H$. pylori-positive and -negative subjects, including miR-21, miRNA-223, miR-146a, miR-155 and let-7 [66-68]. Specifically, CagA triggered aberrant epigenetic silencing of let-7 expression, thus increasing Ras expression [68]. Moreover, miR-370 expression was suppressed by $H$. pylori and CagA inhibited, which led to upregulation of FoxM1, inhibition of $\mathrm{p} 27^{\mathrm{Kip} 1}$ and cell proliferation [69]. In several diseases characterized by chronic inflammation, hypermethylation of the promoter region CpG islands is associated with transcriptional inactivation of tumor suppressor genes. H. pylori has been reported to induce DNA methylation, including Sat $\alpha$ and E-cadherin, leading to the development of gastric cancer [70-73]. Direct adhesion of $H$. pylori to the host cells induces aberrant activation-induced cytidine deaminase expression, resulting in double-strand breaks in host cell genomic sequences [74, 75]. Either introduction of bacterial virulence factors into host cells or induction of inflammatory responses by $\mathrm{H}$. pylori contributes to activation-induced cytidine deaminase expression $[76,77]$.

\section{Chronic Inflammation}

The ongoing chronic inflammation aroused by H. pylori infection is a major step for the initiation and development of gastric cancer. The gastric inflammatory response consists of neutrophils, lymphocytes ( $\mathrm{T}$ and $\mathrm{B}$ cells), plasma cells and macrophages, along with varying degrees of epithelial cell degeneration and injury. H. pylori adhesion to epithelial cells is associated with brisk activation of NF- $\kappa B$ and enhanced level of cytokine interleukin-1b (IL-1b), IL-2, IL-6, IL-8 and TNF- $\alpha$, triggering inflammation in gastric epithelial cells [33]. Cyclooxygenase-2 (COX-2), correlated with gastric carcinogenesis, is also involved in $\mathrm{H}$. pylori-induced inflammatory responses; its expression is most strongly increased in the epithelium of malignant and dysplastic glands. The pro-inflammatory prostaglandin PGE2 is subsequently induced after the upregulation of COX-2. Prostaglandins play an important role in the growth and stimulation of inflammation-associated gastric carcinogenesis [78].

\section{Non-H. pylori Microbiota and Gastric Cancer Development}

Findings from current studies support the role of non-H. pylori microbiota in the development of gastric cancer. Studies with INS-GAS mice have revealed that male mice with intestinal microbiota developed gastric pathology from chronic gastritis to atrophy and dysplasia 
independent of $H$. pylori infection. Besides, the presence of commensal microbiota accelerated the progression to gastric intraepithelial neoplasia, and gastric intraepithelial neoplasia became invasive in $H$. pylori-infected INS-GAS mice [24, 79]. Furthermore, in male INS-GAS mice with $\mathrm{H}$. pylori infection, colonization with artificial mouse intestinal microbiota (Altered Schaedler's Flora, including ASF356 Clostridium species, ASF361 Lactobacillus murinus and ASF519 Bacteroides species) increased the incidence of gastric intraepithelial neoplasia to $69.0 \%$ and promoted the development of cancer $[29,79]$. On the other hand, antibiotic treatments significantly delayed the onset of gastric neoplasia in Helicobacter-free and specific pathogen-free INS-GAS mice [28]. In C57BL/6N mice, pre-infection with gastric microbial populations affects the subsequent immune response to $\mathrm{H}$. pylori, contributing to the diverse outcomes of $H$. pylori infection [25]. After treatment with acid-suppressive drugs, risk of atrophy development is only seen in $H$. pylori-positive subjects [80], while no increased risk has been observed in $H$. pylori-negative individuals or after $H$. pylori eradication, suggesting that the microbiota acquired cannot cause atrophy on their own but could enhance atrophy development together with $H$. pylori [81].

Although studies have proved the existence of complex microbiota in the stomach, the importance of $H$. pylori and the possible impact of the non-H. pylori microbiota in the pathogenesis of atrophic gastritis and gastric cancer are to be further investigated.

\section{Summary}

The incidence of gastric cancer remains highly prevalent in Asia when compared to the West, and $H$. pylori infection is the one gastric cancer risk factor. During the past decades, studies of $H$. pylori have identified several virulence factors contributing to the development of gastric cancer. To date, the application of high-throughput sequencing technology and metagenomics have revealed the existence of a diverse and complex composition of microbiota in the stomach. Additionally, findings from current studies support a role of microbiota in the development of gastric cancer. However, due to the limitation of experimental methods or sample numbers, the properties of gastric microbiota and the mechanisms by which they participate in the genesis of gastric cancer are still not clearly depicted. Moreover, it remains to be understood how the presence of microbiota along with $H$. pylori infection accelerate the progress from gastric disease to cancer.

\section{References}

1 Ferlay J, Soerjomataram I, Ervik M, Dikshit R, Eser S, Mathers C, Rebelo M, Parkin DM, Forman D, Bray F: Cancer Incidence and Mortality Worldwide: IARC CancerBase No. 11 [Internet]. Lyon, International Agency for Research on Cancer, 2013.

2 Herszenyi L, Tulassay Z: Epidemiology of gastrointestinal and liver tumors. Eur Rev Med Pharmacol Sci 2010; 14:249-258.

3 Ferlay J, et al: Cancer incidence and mortality worldwide: sources, methods and major patterns in GLOBOCAN 2012. Int J Cancer 2015;136:E359-E386.

4 Sun XD, et al: Analysis of mortality rate of stomach cancer and its trend in twenty years in China. Zhonghua Zhong Liu Za Zhi 2004;26:4-9.

5 Yang L: Incidence and mortality of gastric cancer in China. World J Gastroenterol 2006;12:17-20.

6 American Cancer Society: What are the risk factors for stomach cancer? Available at: http://www.cancer.org/ cancer/stomachcancer/detailedguide/stomach-cancer-risk-factors.

7 Linz B, et al: An African origin for the intimate association between humans and Helicobacter pylori. Nature 2007;445:915-918.

8 Marshall BJ, Warren JR: Unidentified curved bacilli in the stomach of patients with gastritis and peptic ulceration. Lancet 1984;1:1311-1315. 
9 Patel A, Shah N, Prajapati JB: Clinical application of probiotics in the treatment of Helicobacter pylori infection - a brief review. J Microbiol Immunol Infect 2014;47:429-437.

10 Peek RM Jr, Kuipers EJ: Gained in translation: the importance of biologically relevant models of Helicobacter pylori-induced gastric cancer. Gut 2012;61:2-3.

11 Tlaskalová-Hogenová $\mathrm{H}$, et al: The role of gut microbiota (commensal bacteria) and the mucosal barrier in the pathogenesis of inflammatory and autoimmune diseases and cancer: contribution of germ-free and gnotobiotic animal models of human diseases. Cell Mol Immunol 2011;8:110-120.

12 Wang ZK, Yang YS: Upper gastrointestinal microbiota and digestive diseases. World J Gastroenterol 2013;19: 1541-1550.

13 Bacteria in the stomach. Lancet 1981;2:906-907.

14 Li XX, et al: Bacterial microbiota profiling in gastritis without Helicobacter pylori infection or non-steroidal anti-inflammatory drug use. PLoS One 2009;4:e7985.

15 Ahmed N, et al: Comparative analysis of human gut microbiota by barcoded pyrosequencing. PLoS One 2008; 3:e2836.

16 Bik EM, et al: Molecular analysis of the bacterial microbiota in the human stomach. Proc Natl Acad Sci USA 2006;103:732-737.

17 Seo I, et al: Microbial profile of the stomach: comparison between normal mucosa and cancer tissue in the same patient. J Bacteriol Virol 2014;44:162-169.

18 Delgado S, et al: Microbiological survey of the human gastric ecosystem using culturing and pyrosequencing methods. Microb Ecol 2013;65:763-772.

19 Aviles-Jimenez F, et al: Stomach microbiota composition varies between patients with non-atrophic gastritis and patients with intestinal type of gastric cancer. Sci Rep 2014;4:4202.

20 Eun CS, et al: Differences in gastric mucosal microbiota profiling in patients with chronic gastritis, intestinal metaplasia, and gastric cancer using pyrosequencing methods. Helicobacter 2014;19:407-416.

21 Dicksved J, et al: Molecular characterization of the stomach microbiota in patients with gastric cancer and in controls. J Med Microbiol 2009;58:509-516.

22 Brawner KM, Morrow CD, Smith PD: Gastric microbiome and gastric cancer. Cancer J 2014;20:211-216.

23 Aebischer T, et al: Vaccination prevents Helicobacter pylori-induced alterations of the gastric flora in mice. FEMS Immunol Med Microbiol 2006;46:221-229.

24 Lofgren JL, et al: Lack of commensal flora in Helicobacter pylori-infected INS-GAS mice reduces gastritis and delays intraepithelial neoplasia. Gastroenterology 2011;140:210-220.

25 Rolig AS, et al: The degree of Helicobacter pylori-triggered inflammation is manipulated by preinfection host microbiota. Infect Immun 2013;81:1382-1389.

26 Osaki T, et al: Comparative analysis of gastric bacterial microbiota in Mongolian gerbils after long-term infection with Helicobacter pylori. Microb Pathog 2012;53:12-18.

27 Maldonado-Contreras A, et al: Structure of the human gastric bacterial community in relation to Helicobacter pylori status. ISME J 2011;5:574-579.

28 Lee CW, etal: Combination of sulindac and antimicrobial eradication of Helicobacter pylori prevents progression of gastric cancer in hypergastrinemic INS-GAS mice. Cancer Res 2009;69:8166-8174.

29 Lee CW, et al: Helicobacter pylori eradication prevents progression of gastric cancer in hypergastrinemic INS-GAS mice. Cancer Res 2008;68:3540-3548.

30 Wong BCY, et al: Helicobacter pylori eradication to prevent gastric cancer in a high-risk region of China - a randomized controlled trial. JAMA 2004;291:187-194.

31 Adamsson I, Edlund C, Nord C: Impact of treatment of Helicobacter pylori on the normal gastrointestinal microflora. Clin Microbiol Infect 2000;6:175-177.

32 Bühling A, et al: Influence of anti-Helicobacter triple-therapy with metronidazole, omeprazole and clarithromycin on intestinal microflora. Aliment Pharmacol Ther 2001;15:1445-1452.

33 Israel DA, Peek RM: Pathogenesis of Helicobacter pylori-induced gastric inflammation. Aliment Pharmacol Ther 2001;15:1271-1290.

34 Peek RM Jr, Crabtree JE: Helicobacter infection and gastric neoplasia. J Pathol 2006;208:233-248.

35 Munoz-Moreno L, et al: Inhibitory effects of antagonists of growth hormone-releasing hormone on growth and invasiveness of PC3 human prostate cancer. Int J Cancer 2013;132:755-765.

36 Akopyants NS, et al: Analyses of the cag pathogenicity island of Helicobacter pylori. Mol Microbiol 1998;28: 37-53.

37 Censini S, et al: cag, a pathogenicity island of Helicobacter pylori, encodes type I-specific and disease-associated virulence factors. Proc Natl Acad Sci USA 1996;93:14648-14653.

38 Ohnishi N, et al: Transgenic expression of Helicobacter pylori CagA induces gastrointestinal and hematopoietic neoplasms in mouse. Proc Natl Acad Sci USA 2008;105:1003-1008.

39 Amieva MR, et al: Disruption of the epithelial apical-junctional complex by Helicobacter pylori CagA. Science 2003;300:1430-1434.

40 Saadat I, et al: Helicobacter pylori CagA targets PAR1/MARK kinase to disrupt epithelial cell polarity. Nature 2007;447:330-333.

41 Krueger S, et al: Helicobacter pylori alters the distribution of Z0-1 and p120ctn in primary human gastric epithelial cells. Pathol Res Pract 2007;203:433-444. 
42 Zeaiter Z, et al: Analysis of detergent-resistant membranes of Helicobacter pylori infected gastric adenocarcinoma cells reveals a role for MARK2/Par1b in CagA-mediated disruption of cellular polarity. Cell Microbiol 2008;10:781-794.

43 Murata-Kamiya N, et al: Helicobacter pylori CagA interacts with E-cadherin and deregulates the beta-catenin signal that promotes intestinal transdifferentiation in gastric epithelial cells. Oncogene 2007;26:4617-4626.

44 Suzuki M, et al: Interaction of CagA with Crk plays an important role in Helicobacter pylori-induced loss of gastric epithelial cell adhesion. J Exp Med 2005;202:1235-1247.

45 Rieder G, Fischer W, Haas R: Interaction of Helicobacter pylori with host cells: function of secreted and translocated molecules. Curr Opin Microbiol 2005;8:67-73.

46 Dubois A, Boren T: Helicobacter pylori is invasive and it may be a facultative intracellular organism. Cell Microbiol 2007;9:1108-1116.

47 Weydig C, et al: CagA-independent disruption of adherence junction complexes involves E-cadherin shedding and implies multiple steps in Helicobacter pylori pathogenicity. Exp Cell Res 2007;313:3459-3471.

48 Kwok T, et al: Helicobacter exploits integrin for type IV secretion and kinase activation. Nature 2007; 449: 862-866.

49 Asahi M, et al: Helicobacter pylori CagA protein can be tyrosine phosphorylated in gastric epithelial cells. J Exp Med 2000;191:593-602.

50 Selbach M, et al: Src is the kinase of the Helicobacter pylori CagA protein in vitro and in vivo. J Biol Chem 2002; 277:6775-6778.

51 Stein M, et al: c-Src/Lyn kinases activate Helicobacter pylori CagA through tyrosine phosphorylation of the EPIYA motifs. Mol Microbiol 2002;43:971-980.

52 Higashi H, et al: SHP-2 tyrosine phosphatase as an intracellular target of Helicobacter pylori CagA protein. Science 2002;295:683-686.

53 Higashi H, et al: Helicobacter pylori CagA induces Ras-independent morphogenetic response through SHP-2 recruitment and activation. J Biol Chem 2004;279:17205-17216.

54 Zhao DP, et al: Helicobacter pylori CagA upregulation of CIP2A is dependent on the Src and MEK/ERK pathways. J Med Microbiol 2010;59:259-265.

55 Keates S, et al: Transactivation of the epidermal growth factor receptor by cag+ Helicobacter pylori induces upregulation of the early growth response gene Egr-1 in gastric epithelial cells. Gut 2005;54:1363-1369.

56 Keates S, et al: cag+ Helicobacter pylori induce transactivation of the epidermal growth factor receptor in AGS gastric epithelial cells. J Biol Chem 2001;276:48127-48134.

57 Busiello I, et al: Helicobacter pylori gamma-glutamyltranspeptidase upregulates COX-2 and EGF-related peptide expression in human gastric cells. Cell Microbiol 2004;6:255-267.

58 Tabassam FH, Graham DY, Yamaoka Y: OipA plays a role in Helicobacter pylori-induced focal adhesion kinase activation and cytoskeletal re-organization. Cell Microbiol 2008;10:1008-1020.

59 Siegfried JM, et al: Combining the multitargeted tyrosine kinase inhibitor vandetanib with the antiestrogen fulvestrant enhances its antitumor effect in non-small cell lung cancer. J Thorac Oncol 2012; 7:485-495.

60 Nagy TA, et al: Helicobacter pylori regulates cellular migration and apoptosis by activation of phosphatidylinositol 3-kinase signaling. J Infect Dis 2009;199:641-651.

61 Maeda S, et al: Analysis of apoptotic and antiapoptotic signalling pathways induced by Helicobacter pylori. Gut 2002;50:771-778.

62 Suzuki M, et al: Helicobacter pylori CagA phosphorylation-independent function in epithelial proliferation and inflammation. Cell Host Microbe 2009;5:23-34.

63 Kaparakis M, et al: Bacterial membrane vesicles deliver peptidoglycan to NOD1 in epithelial cells. Cell Microbiol 2010;12:372-385.

64 Nakayama M, et al: Helicobacter pylori VacA-induced inhibition of GSK3 through the PI3K/Akt signaling pathway. J Biol Chem 2009;284:1612-1619.

65 Franco AT, et al: Regulation of gastric carcinogenesis by Helicobacter pylori virulence factors. Cancer Res 2008; 68:379-387.

66 Matsushima K, et al: MicroRNA signatures in Helicobacter pylori-infected gastric mucosa. Int J Cancer 2011; 128:361-370.

67 Liu Z, et al: Up-regulated microRNA-146a negatively modulate Helicobacter pylori-induced inflammatory response in human gastric epithelial cells. Microbes Infect 2010;12:854-863.

68 Hayashi Y, et al: CagA mediates epigenetic regulation to attenuate let-7 expression in Helicobacter pylorirelated carcinogenesis. Gut 2013;62:1536-1546.

69 Feng YM, et al: FoxM1 is overexpressed in Helicobacter pylori-induced gastric carcinogenesis and is negatively regulated by miR-370. Mol Cancer Res 2013;11:834-844.

70 Yoshida T, et al: Altered mucosal DNA methylation in parallel with highly active Helicobacter pylori-related gastritis. Gastric Cancer 2013;16:488-497.

71 Cheng AS, et al: Helicobacter pylori causes epigenetic dysregulation of FOXD3 to promote gastric carcinogenesis. Gastroenterology 2013;144:122-133.e9.

72 Saito M, et al: The accumulation of DNA demethylation in Sat alpha in normal gastric tissues with Helicobacter pylori infection renders susceptibility to gastric cancer in some individuals. Oncol Rep 2012;27:1717-1725.

73 Huang FY, et al: Helicobacter pylori induces promoter methylation of E-cadherin via interleukin-1beta activation of nitric oxide production in gastric cancer cells. Cancer 2012;118:4969-4980. 
74 Uehara T, et al: H. pylori infection is associated with DNA damage of Lgr5-positive epithelial stem cells in the stomach of patients with gastric cancer. Dig Dis Sci 2013;58:140-149.

75 Toller IM, et al: Carcinogenic bacterial pathogen Helicobacter pylori triggers DNA double-strand breaks and a DNA damage response in its host cells. Proc Natl Acad Sci USA 2011;108:14944-14949.

76 Shimizu T, et al: Accumulation of somatic mutations in TP53 in gastric epithelium with Helicobacter pylori infection. Gastroenterology 2014;147:407-417.e3.

77 Marusawa $\mathrm{H}$, Chiba T: Helicobacter pylori-induced activation-induced cytidine deaminase expression and carcinogenesis. Curr Opin Immunol 2010;22:442-447.

78 Shao Y, et al: Helicobacter pylori infection, gastrin and cyclooxygenase-2 in gastric carcinogenesis. World J Gastroenterol 2014;20:12860-12873.

79 Lertpiriyapong K, et al: Gastric colonisation with a restricted commensal microbiota replicates the promotion of neoplastic lesions by diverse intestinal microbiota in the Helicobacter pylori INS-GAS mouse model of gastric carcinogenesis. Gut 2014;63:54-63.

80 Naylor G, Axon A: Role of bacterial overgrowth in the stomach as an additional risk factor for gastritis. Can J Gastroenterol 2003;17(suppl B):13B-17B.

81 Engstrand L, Lindberg M: Helicobacter pylori and the gastric microbiota. Best Pract Res Clin Gastroenterol 2013;27:39-45.

82 Zhang JF, et al: Ectopic expression of guanylyl cyclase C and endogenous ligand guanylin correlates significantly with Helicobacter pylori infection in gastric carcinogenesis. Med Oncol 2012;29:1748-1757. 